\title{
Design Methodology for Coplanar Axes Line Gear with Controllable Sliding Rate
}

\author{
Yangzhi Chen - Zheng Li - Xiongdun Xie - Yueling Lyu* \\ South China University of Technology, China
}

\begin{abstract}
Line gear (LG) pair is a novel gear mechanism via point contact meshing on the basis of space curve meshing theory. In this paper, aiming to control the sliding rate, related to the shape of the contact curves of LGs, the driving contact curve of a coplanar axes LG pair was extended from a circular helix to a conic helix with variable cone angle, and the driven contact curve was subsequently obtained based on the space curve meshing theory. A new type of line tooth, of which two contact curves lie on each side, was proposed for forward-reverse transmission without backlash. The tooth surface is formed by a normal tooth profile moving along the contact curve and the tooth thickness auxiliary curve. The formulae of the contact curves, tooth thickness auxiliary curves, and spatial cylindrical tooth surfaces of the new type of line tooth are derived, which theoretically provides a foundation for the standardized production of $L G$ pairs in industry. The calculation examples of the $L G$ pairs show that the sliding rate can be reduced by using a conic helix as the driving contact curve. The results of the kinematics experiment show that the LG pair designed by the methodology could be capable of achieving forward-reverse transmission without backlash and with a controllable sliding rate.
\end{abstract}

Keywords: line gear, sliding rate, contact curve, tooth surface, non-backlash, forward-reverse transmission

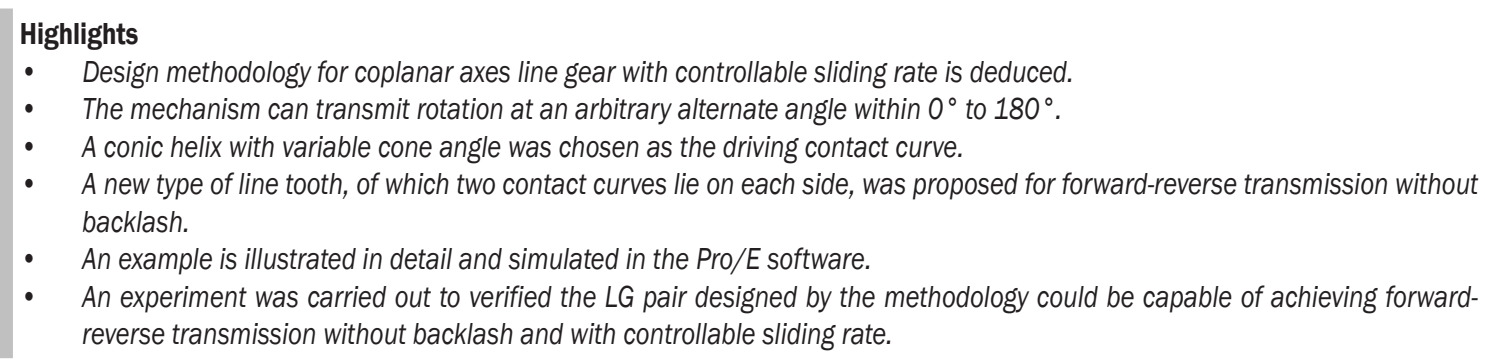

\section{O INTRODUCTION}

In 1733, Camus' Theorem [1] was proposed for the conjugation of tooth profiles. In 1956, Beam [2] first proposed the so-called 'Beveloid gears', which can be adopted for power transmission between nearlyparallel shafts and allow for adjustment of the gearing backlash, following which many studies detailed their geometry, design and manufacturing [3] to [5]. In 1962, Baxter [6] analysed the basic geometric and tooth contact of hypoid gears. In the 1980s, Litvin et al. [7] systematically proposed the gear geometry theory. In recent decades, the design and manufacturing of gear pairs transmitting rotations between intersecting shafts have been a topic of research [8] to [10].

The line gear (LG) pair, also called a 'space curve meshing wheel' (SCMW) in studies before 2014, proposed by Chen [11], is a novel transmission mechanism via point contact meshing on the basis of space curve meshing theory. According to the basic theory of LG, the motion at the meshing point should satisfy the following equation [12]:

$$
v_{12} \times \beta=0,
$$

where, $\boldsymbol{v}_{\mathbf{1 2}}$ is the relative velocity at the meshing point between the driving contact curve and the driven contact curve, and $\boldsymbol{\beta}$ is the unit principal normal vector of the driving contact curve at the meshing point.

Compared with traditional gear, LG has characteristics of compact space, large transmission ratio and flexible design theory [11]. There is no undercut phenomenon on the line gear, and the minimum number of the line teeth of an line gear equals 1. It could be flexible to design for the application of the LGs to transmissions with perpendicular shafts [13], intersecting shafts [14] or skew shafts [15]. The driving contact curve of the coplanar axes of the LG pair is a circular helix, satisfying the condition of contact ratio [16], and a conjugated driven contact curve can be obtained through the coordinate system transformation based on space curve meshing equation Eq. (1), which may be a circular helix, a conic helix or a planar Archimedean spiral. An LG pair consists of a driving $L G$ and a driven LG. The driving line teeth and the driven line teeth are uniformly distributed around the basal wheels of the driving LG and the driven LG, respectively [15]. There are mainly two forms of the line-tooth of LG, one of which is cantilever structural 
form with a circular profile, and the other is that its line tooth is constructed by a convex or a concave arc profile radially attached to the wheel body. The cantilever structural tooth is the simplest structure in previously published papers. The line tooth radially attached to the wheel body is suitable for the manufacturing process of cutting materials such as CNC machining, and be of better bearing capacity [17] and [18].

The sliding rate of a gear pair, used for describing the relative movement trend of each gear at the meshing point, is a critical impacting factor on the transmission quality [19]. The relative sliding between two tooth surfaces might bring about negative influences such as surface wear and frictional loss, so the allowable value of the sliding rate depends on different working conditions such as load, speed, lubrication condition and so on [20] and [21]. If a good elasto-hydrodynamic-lubrication (EHL) oil film is formed at a certain relative sliding speed, the surface wear will be eliminated and transmission efficiency will be increased [22] and [23], at which circumstance the main failure form of gears is tooth surface fatigue pitting. Therefore, for the gear transmission, the research result on sliding rate is the basis of gear lubrication and failure analysis.

The sliding rate of the LG pair depends on the relative position parameters and the meshing radius of the LG pair. Aiming to decrease the sliding rate and reduce the gear wear, the design process usually follows the position parameter selection criterion in which the sliding rate equals zero at the midpoint of the contact curves [24].

In this paper, with the aim of modifying the sliding rate of the LG pair, a conic helix with variable cone angle was chosen as the driving contact curve. Then, the equation forms of the driving contact curve and the driven contact curve in the respective followup coordinate systems were unified. A new type of the line-tooth of LG was subsequently designed, which could be capable of achieving forward-reverse transmission without backlash. There are two contact curves that lie on each side of one line tooth. Two normal convex arc tooth profiles are established in the normal sections of two contact curves at any meshing point to form a line tooth entity.

\section{DESIGN METHODS}

\subsection{Design Formulae of a Pair of Coplanar Axes LGs}

The axes of the driving LG and the driven LG are in the same plane, covering the parallel axes and arbitrary angle intersecting axes. The coordinate systems are established, as shown in Fig. 1. The coordinate systems $o_{0}-x_{0} y_{0} z_{0}$ and $o_{1}-x_{1} y_{1} z_{1}$ are the fixed coordinate system and the follow-up coordinate system of a driving LG, respectively. The coordinate systems $o_{p}-x_{p} y_{p} z_{p}$ and $o_{2}-x_{2} y_{2} z_{2}$ are the fixed coordinate system and the follow-up coordinate system of a driven $L G$, respectively. The driving $L G$ rotates around $z_{0}\left(z_{1}\right)$-axis and the driven LG rotates around $z_{2}\left(z_{p}\right)$-axis. The shaft angle between $z_{0}\left(z_{1}\right)$ axis and $z_{2}\left(z_{p}\right)$-axis is denoted as $\theta_{0}, \theta_{0} \in[0, \pi)$. With the aim of avoiding overlap between two shafts of LG pair, define $o_{0} o_{p}=a$.

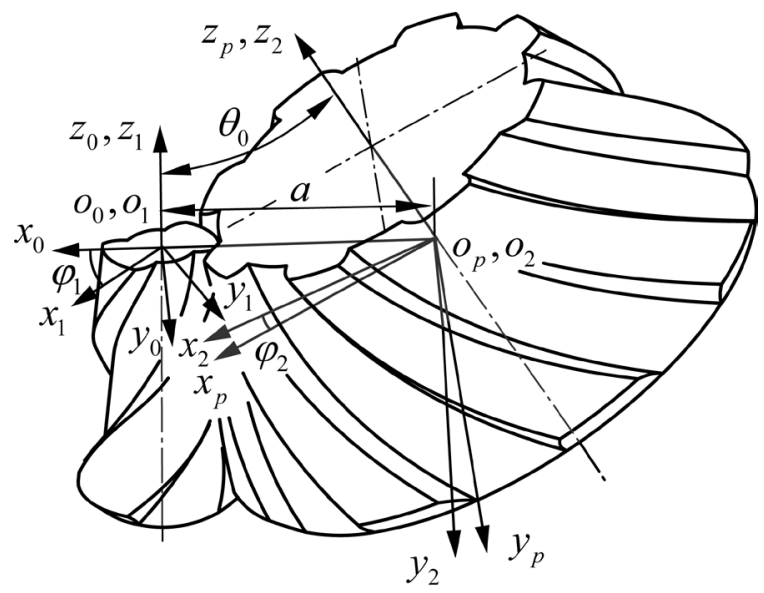

Fig. 1. The coordinate systems of a pair of coplanar axes LGs

The transformation matrixes for the coordinate systems in the design process of the coplanar axes LG are as Eqs. (2) to (4).

$$
\begin{gathered}
\boldsymbol{M}_{p 0}=\left[\begin{array}{cccc}
\cos \theta_{0} & 0 & -\sin \theta_{0} & a \cos \theta_{0} \\
0 & 1 & 0 & 0 \\
\sin \theta_{0} & 0 & \cos \theta_{0} & a \sin \theta_{0} \\
0 & 0 & 0 & 1
\end{array}\right], \\
\boldsymbol{M}_{01}=\left[\begin{array}{cccc}
\cos t & -\sin t & 0 & 0 \\
\sin t & \cos t & 0 & 0 \\
0 & 0 & 1 & 0 \\
0 & 0 & 0 & 1
\end{array}\right] \\
\boldsymbol{M}_{2 p}=\left[\begin{array}{cccc}
\cos \frac{t}{i} & -\sin \frac{t}{i} & 0 & 0 \\
\sin \frac{t}{i} & \cos \frac{t}{i} & 0 & 0 \\
0 & 0 & 1 & 0 \\
0 & 0 & 0 & 1
\end{array}\right]
\end{gathered}
$$


where, $t$ is an independent parameter of the contact curves and $i$ is the transmission ratio.

The equation of a driving contact curve in $o_{1}-x_{1} y_{1} z_{1}$ is given as Eq. (5).

$$
\boldsymbol{R}_{1}^{1}=\left\{\begin{array}{l}
x_{1}=-\left(m_{1}+n_{1} t \sin \theta_{1}\right) \cos t \\
y_{1}=\left(m_{1}+n_{1} t \sin \theta_{1}\right) \sin t, \\
z_{1}=-n_{1} t \cos \theta_{1}
\end{array},\right.
$$

where, $\theta_{1}$ is half cone angle, $m_{1}$ and $n_{1}$ are conical parameters. When $\theta_{1}$ equals to 0 , the driving contact curve is a circular helix. Under the following conditions for the value of the parameter $\theta_{1}$, $\theta_{1} \in\left(0, \theta_{0}\right]$ and $\theta_{1} \neq 90^{\circ}$, the driving contact curve is a common conic helix. When $\theta_{1}$ equals to $90^{\circ}$, the driving contact curve is a planar Archimedean spiral.

From Eqs. (2) to (5), the equation of a driven contact curve in $o_{2}-x_{2} y_{2} z_{2}$, conjugated with the given driving contact curve above, is reflected as follows.

$$
\begin{aligned}
& \boldsymbol{R}_{2}^{2}=\boldsymbol{M}_{2 p} \cdot \boldsymbol{M}_{p 0} \cdot \boldsymbol{M}_{01} \cdot \boldsymbol{R}_{1}^{1} \\
& =\left\{\begin{array}{l}
x_{2}=\left[\left(a-m_{1}\right) \cos \theta_{0}+n_{1} t \sin \left(\theta_{0}-\theta_{1}\right)\right] \cos \frac{t}{i} \\
y_{2}=\left[\left(a-m_{1}\right) \cos \theta_{0}+n_{1} t \sin \left(\theta_{0}-\theta_{1}\right)\right] \sin \frac{t}{i} \\
z_{2}=-n_{1} t \cos \left(\theta_{0}-\theta_{1}\right)+\left(a-m_{1}\right) \sin \theta_{0}
\end{array}\right.
\end{aligned}
$$

Similar to the driving contact curve, the driven contact curve may be a circular helix, a conic helix or a planar Archimedean spiral.

Eqs. (5) and (6) with different parameters $\theta_{0}$ and $\theta_{1}$ can generally be used to describe a circular helix, a conic helix and a planar Archimedean spiral. Therefore, in geometry, an undifferentiated form of mathematical expressions of the contact curves of an LG pair exists, which can generally be used in the research of the line-tooth entity construction and machining method of the driving LG and the driven LG.

Referring to $\boldsymbol{R}_{1}^{1}$, aiming to eliminate the constant $\left(a-m_{1}\right) \sin \theta_{0}$ in $\boldsymbol{R}_{2}^{2}$, the equation of the driven contact curve in a new follow-up coordinate system $o_{2^{\prime}}-x_{2^{\prime}} y_{2^{\prime}} z_{2^{\prime}}$, which is formed by moving the coordinate system $o_{2}-x_{2} y_{2} z_{2}$ along $z_{2}$-axis by the distance $\left(a-m_{1}\right) \sin \theta_{0}$, is expressed as Eq. (7).

$$
\boldsymbol{R}_{2}^{2 \prime}=\left\{\begin{array}{l}
x_{2}=\left[\left(a-m_{1}\right) \cos \theta_{0}+n_{1} t \sin \left(\theta_{0}-\theta_{1}\right)\right] \cos \frac{t}{i} \\
y_{2}=\left[\left(a-m_{1}\right) \cos \theta_{0}+n_{1} t \sin \left(\theta_{0}-\theta_{1}\right)\right] \sin \frac{t}{i} \cdot(7) \\
z_{2}=-n_{1} t \cos \left(\theta_{0}-\theta_{1}\right)
\end{array}\right.
$$

The transformation matrix between two coordinate systems, from $o_{2},-x_{2} y_{2} z_{2}$, to $o_{2}-x_{2} y_{2} z_{2}$, is as Eq. (8), which is formed by moving the coordinate system:

$$
\boldsymbol{M}_{22^{\prime}}=\left[\begin{array}{cccc}
1 & 0 & 0 & 0 \\
0 & 1 & 0 & 0 \\
0 & 0 & 1 & \left(a-m_{1}\right) \sin \theta_{0} \\
0 & 0 & 0 & 1
\end{array}\right] .
$$

After unifying the coordinate systems $o_{1}-x_{1} y_{1} z_{1}$ and $o_{2}, x_{2^{\prime}} y_{2^{\prime}} z_{2^{\prime}}$ into the coordinate system $o_{m}-x_{m} y_{m} z_{m}$, which can generally be used to describe the driving contact curve and the driven contact curve, from Eqs. (5) and (7), the undifferentiated form of the contact curves of an LG pair can be written as Eq. (9).

$$
\boldsymbol{R}_{j 1}^{m}=\left\{\begin{array}{l}
x_{j}=k_{j}\left(m_{j}+n_{j} t_{j} \sin \theta_{j}\right) \cos t_{j} \\
y_{j}=\left(m_{j}+n_{j} t_{j} \sin \theta_{j}\right) \sin t_{j} \\
z_{j}=-n_{j} t_{j} \cos \theta_{j} \\
(j=1,2)
\end{array},\right.
$$

where, $\boldsymbol{R}_{11}^{m}$ represents a driving contact curve and $\boldsymbol{R}_{21}^{m}$ represents a driven contact curve. $t_{j}$ are independent parameters, $t_{j} \in\left[t_{j s}, t_{j e}\right] . t_{j s}$ and $t_{j e}$ are starting values and ending values for meshing points, respectively. $\theta_{j}$ are half cone angles, $m_{j}$ and $n_{j}$ are conical parameters. $k_{j}$ represents a parameter of the hand of the spiral. When $k_{j}$ equals 1 , the conic helix is left-handed, and when $k_{j}$ equals -1 , it is right-handed.

The equations of a driving contact curve and a driven contact curve are uniquely determined by the parameters $\theta_{j}, m_{j}, n_{j}, k_{j}$. The parameters of a driving contact curve, a driven contact curve and the relative position parameters should meet the following mathematical conditions:

$$
\left\{\begin{array}{c}
\frac{t_{1}}{t_{2}}=\frac{n_{2}}{n_{1}}=i \\
m_{1}+m_{2} \sec \theta_{0}=a \\
\theta_{1}+\theta_{2}=\theta_{0} \\
-k_{1}=k_{2}=1
\end{array}\right.
$$

A driving contact curve and a driven contact curve of an LG pair are uniquely determined by parameters required, including relative position parameters $a$ and $\theta_{0}$, transmission ratio $i$, conical parameters $m_{1}$ and $n_{1}$, half cone angle $\theta_{1}$, starting value $t_{1 s}$ and ending value $t_{1 e}$ for meshing point of a driving contact curve.

The values of parameters $t_{1 s}$ and $t_{1 e}$ should satisfy the condition of contact ratio [16]. For a special 
condition of that $\theta_{0}$ equals to $90^{\circ}$, the value of the parameter $m_{2}$ can be selected on request.

\subsection{Construction of Line Tooth}

The motion transmission for an LG pair exists via two contact curves on the driving LG and the driven LG, respectively, which are expressed as Eq. (9). The contact curves lie on the line teeth entity with a spatial cylindrical surface [18]. The driving line teeth and the driven line teeth are uniformly distributed around the cylindrical or conical wheel bodies of the driving LG and the driven LG.

Aiming to realize the non-backlash forwardreverse transmission, the number of contact curves on each line tooth equals two, including the first contact curve $\boldsymbol{R}_{j 1}^{m}$ and the second contact curve $\boldsymbol{R}_{j 2}^{m}$. That is, the driving contact curves in one line tooth of the driving LG include the first contact curve of the driving LG $\boldsymbol{R}_{11}^{m}$ and the second contact curve of the driving LG $\boldsymbol{R}_{12}^{m}$, and the driven contact curves in one line tooth of the driven LG include the first contact curve of the driven LG $\boldsymbol{R}_{21}^{m}$ and the second contact curve of the driven LG $\boldsymbol{R}_{22}^{m}$. When the driving LG rotates forward, $\boldsymbol{R}_{11}^{m}$ it engages with $\boldsymbol{R}_{21}^{m}$. And when it rotates in reverse, $\boldsymbol{R}_{12}^{m}$ it engages with $\boldsymbol{R}_{22}^{m}$.

$\boldsymbol{R}_{11}^{m}$ and $\boldsymbol{R}_{21}^{m}$ are expressed as Eq. (9). $\boldsymbol{R}_{12}^{m}$ is obtained by rotating $\boldsymbol{R}_{11}^{m}$ an angle of $\varphi_{1 F R}$ around the axis of the driving LG clockwise, and $\boldsymbol{R}_{22}^{m}$ is obtained by rotating $\boldsymbol{R}_{21}^{m}$ an angle of $\varphi_{2 F R}$ around the axis of the driven LG counter-clockwise. Because the line teeth are uniformly distributed in the circumferential direction of the wheel body, $\boldsymbol{R}_{j 1 n e}^{m}$, the first contact curve that lies on the next line tooth adjacent to the second contact curve $\boldsymbol{R}_{j 2}^{m}$, can be obtained by rotating $\boldsymbol{R}_{j 1}^{m}$ an angle of $2 \pi / N_{j}$ around the axis of the LG, where $N_{1}$ is tooth number of the driving LG, and $N_{2}$ is tooth number of the driven LG, as shown in Fig. 2.

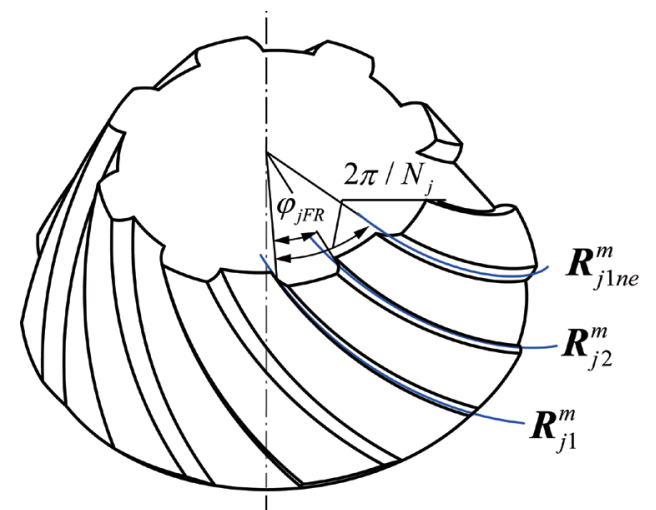

Fig. 2. The schematic diagram of contact curves
The transformation matrixes for coordinate systems involved are as Eqs. (11) and (12).

$$
\begin{gathered}
\boldsymbol{M}_{j 2, j 1}^{m}=\left[\begin{array}{cccc}
\cos \varphi_{j F R} & -k_{j} \sin \varphi_{j F R} & 0 & 0 \\
k_{j} \sin \varphi_{j F R} & \cos \varphi_{j F R} & 0 & 0 \\
0 & 0 & 1 & 0 \\
0 & 0 & 0 & 1
\end{array}\right], \\
\boldsymbol{M}_{j 1 n e, j 1}^{m}=\left[\begin{array}{cccc}
\cos \frac{2 \pi}{N_{j}} & -k_{j} \sin \frac{2 \pi}{N_{j}} & 0 & 0 \\
k_{j} \sin \frac{2 \pi}{N_{j}} & \cos \frac{2 \pi}{N_{j}} & 0 & 0 \\
0 & 0 & 1 & 0 \\
0 & 0 & 0 & 1
\end{array}\right] .
\end{gathered}
$$

The values of the parameters $\varphi_{1 F R}$ and $\varphi_{2 F R}$ determine the relative positions of the first contact curve and the second contact curve of the LGs. The second contact curves of the driving LG and the driven LG are also a couple of conjugated curves satisfying the meshing condition, which is expressed as Eq. (13).

$$
\boldsymbol{M}_{22} \cdot \boldsymbol{R}_{22}^{m}=\boldsymbol{M}_{2 p} \cdot \boldsymbol{M}_{p 0} \cdot \boldsymbol{M}_{01} \cdot \boldsymbol{R}_{12}^{m} .
$$

Furthermore, the values of the parameters $\varphi_{1 F R}$ and $\varphi_{2 F R}$ determine the tooth thickness and the space width, which can be defined in the transverse plane, normal plane. and axial section. According to the meshing theory of LG, while the driving LG rotates forward or reverses at the same position, the two meshing points located respectively at the first contact curve or the contact curve of a line tooth lie on the same axial section of the LG. Therefore, the line-tooth thickness and the space width of LG are defined in the axial section, i.e., the line-tooth thickness and the space width are along the direction of a straight generatrix of a suppositional conical surface, a cylindrical surface or a plane, where the contact curves lie on. The line tooth thickness and the space width are two constant values, and they are independent of the position of the meshing point. The line tooth thickness $s_{j}$ of LG is defined as the distance between two intersection points, one of which is the intersection point of $\boldsymbol{R}_{j 1}^{m}$ and any one axial section, the other is the intersection point of $\boldsymbol{R}_{j 2}^{m}$ and the axial section mentioned above. The space width $e_{j}$ is defined as the distance between two intersection points, one of which is the intersection point of $\boldsymbol{R}_{j 2}^{m}$ and any one axial section, the other is the intersection 
point of $\boldsymbol{R}_{j 1 n e}^{m}$ and the axial section mentioned above, as shown in Fig.3.

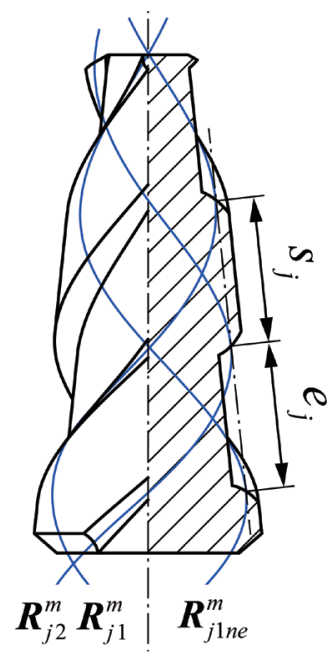

Fig. 3. The schematic diagram of line tooth thickness and the space width in axial section

From Eqs. (8), (11) and (12), the parameters $s_{j}$ and $e_{j}$ are derived.

$$
s_{j}=n_{j} \varphi_{j F R}, \quad e_{j}=n_{j}\left(2 \pi / N_{j}-\varphi_{j F R}\right) .
$$

An LG pair with non-backlash forward-reverse transmission should satisfy the correct meshing condition, that is, the line-tooth thickness of the driving $L G$ is equal to the space width of the driven LG, and the space width of the driving LG is equal to the line-tooth thickness of the driven LG. The correct meshing condition of an LG pair is as Eq. 15.

$$
\left\{\begin{array}{l}
s_{1}=e_{2} \\
s_{2}=e_{1}
\end{array} .\right.
$$

From Eqs. (8), (11) to (15), the second contact curves of the driving LG and the driven LG meeting the correct meshing condition were obtained

$$
\boldsymbol{R}_{j 2}^{m}=\left\{\begin{array}{l}
x_{j}=k_{j} m_{j}^{\prime} \cos \left(t_{j}+\frac{\pi}{N_{j}}\right) \\
y_{j}=m_{j}^{\prime} \sin \left(t_{j}+\frac{\pi}{N_{j}}\right), \quad(j=1,2), \\
z_{j}=-n_{j} t_{j} \cos \theta_{j}
\end{array}\right.
$$

where $m_{j}{ }^{\prime}=m_{j}+n_{j} t_{j} \sin \theta_{j}$.

A line tooth in this paper is composed of three surfaces, including $\Sigma_{j 1}^{m}, \Sigma_{j 2}^{m}$ and $\Sigma_{j 3}^{m}$. $\Sigma_{j 1}^{m}$ and $\Sigma_{j 2}^{m}$ are two parts of spatial cylindrical surfaces located at each side of the line-tooth. The contact curves $\boldsymbol{R}_{j 1}^{m}$ and $\boldsymbol{R}_{j 2}^{m}$ lie on $\Sigma_{j 1}^{m}$ and $\Sigma_{j 2}^{m}$, respectively. The normal tooth profile is located in the normal section of the contact curve at any meshing point. The normal tooth profile is a section of invariant arc with the radius of $r$, which intersects with the contact curve. While the normal tooth profile moves along the contact curve, which takes a role of a generatrix, the continuous spatial cylindrical tooth surfaces are formed, i.e. the spatial cylindrical tooth surfaces $\Sigma_{j 1}^{m}$ is formed by a section of arc moving along the $\boldsymbol{R}_{j 1}^{m}$, which takes a role of a generatrix, and the $\Sigma_{j 2}^{m}$ is formed by a section of arc moving along the $\boldsymbol{R}_{j 2}^{m}$ in the same principal. $\Sigma_{j 3}^{m}$ is the upper surface. If $\theta_{j}$ equals to $0, \Sigma_{j 3}^{m}$ is a cylindrical surface. If $\theta_{j}$ equals to $90^{\circ}, \Sigma_{j 3}^{m}$ is a plane. Under following conditions for the value of the parameter $\theta_{1}, \theta_{1} \in\left(0, \theta_{0}\right], \Sigma_{j 3}^{m}$ is a conical surface.

Because the contact curves are spatial curves, for the definition of the positions of $\Sigma_{j 1}^{m}$ and $\Sigma_{j 2}^{m}$, which must satisfy the conditions of none meshing interference [18], the second constraint should be requested. Therefore, the tooth thickness auxiliary curves are defined as the trajectories of arc centres of normal tooth profiles. There are two functions of the tooth thickness auxiliary curves, one of which is to limit the tooth profile constantly perpendicular to a tangent vector of the contact curve, and the other is to keep the arc radius $r$. The tooth thickness auxiliary curves comprise the first tooth thickness auxiliary curve $\boldsymbol{R}_{j 1 c}^{m}$ and the second tooth thickness auxiliary curve $\boldsymbol{R}_{j 2 c}^{m}$, lying between the first contact curve and the second contact curve, and the first (or second) tooth thickness auxiliary curve is shifted $\overrightarrow{\boldsymbol{M M}_{1}}$ (or $\overrightarrow{\boldsymbol{M M}_{2}}$ ) from every point on the first (or second) contact curve, as shown in Fig. 4. The spatial cylindrical tooth surfaces $\Sigma_{j 1}^{m}$ and $\Sigma_{j 2}^{m}$ are formed by the two sections of normal tooth profiles moving along the corresponding contact curves and the tooth thickness auxiliary curves respectively.

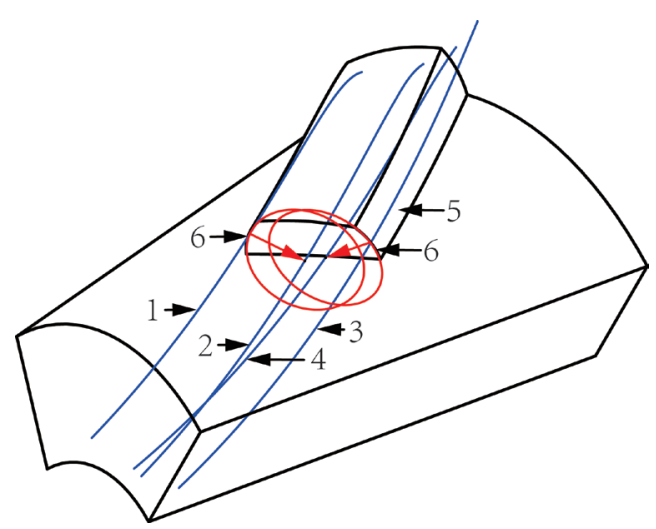

Fig. 4. The schematic diagram of the construction of the line-tooth 
The directions of $\overrightarrow{\boldsymbol{M M}_{1}}$ and $\overrightarrow{\boldsymbol{M M}_{2}}$ are defined to be perpendicular to the tangent vector of the corresponding contact curves, respectively, so that the tooth thickness auxiliary curves can limit the normal tooth profile constantly perpendicular to the tangent vector of the contact curve.

The coordinate system $o_{q}-x_{q} y_{q} z_{q}$, a fixed coordinate system, is an auxiliary coordinate system that concisely describes the coordinates of any meshing point. The $z_{q}$-axis is coincident with the meshing line, the trajectory of a meshing point in the fixed coordinate system, and the $x_{q}$-axis and the shaft of LG intersect at a right angle. The transformation matrix from the coordinate system $o_{q}-x_{q} y_{q} z_{q}$ to the coordinate system $o_{m}-x_{m} y_{m} z_{m}$ is as Eq. (17).

$$
\begin{aligned}
& \boldsymbol{M}_{m q}=\left[\begin{array}{cc}
-k_{j} \cos \theta_{j} \cos t_{j} & -k_{j} \sin \theta_{j} \\
-\cos \theta_{j} \sin t_{j} & \cos t_{j} \\
-\sin \theta_{j} & 0 \\
0 & 0
\end{array}\right. \\
& \left.\begin{array}{cc}
-k_{j} \sin \theta_{j} \cos t_{j} & k_{j} m_{j} \cos t_{i} \\
-\sin \theta_{j} \sin t_{j} & m_{j} \sin t \\
\cos \theta_{j} & 0 \\
0 & 1
\end{array}\right] .
\end{aligned}
$$

In the coordinate system $o_{q}-x_{q} y_{q} z_{q}, \overrightarrow{\boldsymbol{M M}_{1}}$ and $\overrightarrow{\boldsymbol{M M}_{2}}$ are perpendicular to a tangent vector $\boldsymbol{\alpha}$ of the corresponding contact curves at the meshing point $M$, that is, $\overrightarrow{\boldsymbol{M M}_{1}}$ and $\overrightarrow{\boldsymbol{M M}_{2}}$ both lie on the normal plane $\pi$ of the corresponding contact curves at meshing point $M$. Fig. 5. shows the directions of $\overrightarrow{\boldsymbol{M M}_{1}}$ and $\overrightarrow{\boldsymbol{M M}_{2}}$, starting from the meshing point $M$ on the first contact curve and the second contact curve, respectively.

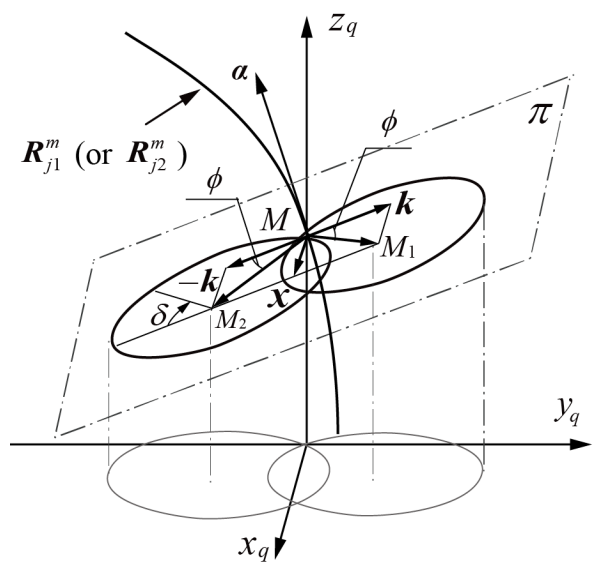

Fig. 5. The schematic diagram of $\overrightarrow{\boldsymbol{M M}_{1}}$ and $\overrightarrow{\boldsymbol{M M}_{2}}$
In the coordinate system $o_{q}-x_{q} y_{q} z_{q}$, the tangent vector $\boldsymbol{\alpha}$ at the meshing point is expressed as:

$$
\alpha=\left[\begin{array}{c}
0 \\
\frac{m_{j}^{\prime}}{\sqrt{n_{j}^{2}+m_{j}^{\prime 2}}} \\
-\frac{n_{j}}{\sqrt{n_{j}^{2}+m_{j}^{\prime 2}}}
\end{array}\right] .
$$

The direction of the vector $\boldsymbol{x}=\left[\begin{array}{lll}1 & 0 & 0\end{array}\right]^{\mathrm{T}}$ is utilized to indicate the radially extending of line tooth. The vector $\boldsymbol{k}$ in the normal plane is perpendicular to the vector $\boldsymbol{x}$, which is utilized to indicate the circumferentially extending of line tooth and is expressed as:

$$
\boldsymbol{k}=\boldsymbol{x} \times \boldsymbol{\alpha}=\left[\begin{array}{c}
0 \\
\frac{n_{j}}{\sqrt{n_{j}^{2}+m_{j}^{\prime 2}}} \\
\frac{m_{j}^{\prime}}{\sqrt{n_{j}^{2}+m_{j}^{\prime 2}}}
\end{array}\right] .
$$

In the coordinate system $o_{q}-\underline{x}_{q} y_{q} z_{q}$, the analytical expressions of $\overrightarrow{\boldsymbol{M M}_{1}}$ and $\overrightarrow{\boldsymbol{M M}_{2}}$ can be derived.

$$
\overrightarrow{\boldsymbol{M} \boldsymbol{M}_{1(2)}}=r(\boldsymbol{x} \sin \phi \pm \boldsymbol{k} \cos \phi)=r\left[\begin{array}{c}
\sin \phi \\
\pm \frac{n_{j} \cos \phi}{\sqrt{m_{j}^{\prime 2}+n_{j}^{2}}} \\
\pm \frac{m_{j}^{\prime} \cos \phi}{\sqrt{m_{j}^{\prime 2}+n_{j}^{2}}}
\end{array}\right],
$$

where $\phi$ is a parameter determining the positions of $\Sigma_{j 1}^{m}$ and $\Sigma_{j 2}^{m}$. Usually $\phi$ is equal to $30^{\circ}$.

The analytical expressions of the tooth surfaces $\Sigma_{j 1}^{m}$ and $\Sigma_{j 2}^{m}$ are achieved. $\delta$ is a parameter to form the surfaces. 


$$
\begin{aligned}
\Sigma_{j 1(j 2)}= & r(\boldsymbol{x} \sin \phi \pm \boldsymbol{k} \cos \phi)+r(\boldsymbol{x} \sin \delta \pm \boldsymbol{k} \cos \delta) \\
& =r\left[\begin{array}{c}
\sin \phi+\sin \delta \\
\pm \frac{n_{j}(\cos \phi+\cos \delta)}{\sqrt{m_{j}^{\prime 2}+n_{j}^{2}}} \\
\pm \frac{m_{j}^{\prime}(\cos \phi+\cos \delta)}{\sqrt{m_{j}^{\prime 2}+n_{j}^{2}}}
\end{array}\right]
\end{aligned}
$$$$
\begin{aligned}
& \Sigma_{j 1}^{m}=\boldsymbol{R}_{j 1}^{m}+\overrightarrow{\boldsymbol{M M}_{1}^{m}}+\boldsymbol{r}_{j 1}, \\
& \Sigma_{j 2}^{m}=\boldsymbol{R}_{j 2}^{m}+\boldsymbol{M}_{j 2 j 1}^{m} \cdot\left(\overrightarrow{\boldsymbol{M M}_{2}^{m}}+\boldsymbol{r}_{j 2}\right),
\end{aligned}
$$

where

Back to the coordinate system $o_{m}-x_{m} y_{m} z_{m}$, by utilizing the transformation matrix $\boldsymbol{M}_{m q}$, the analytical expressions of $\overrightarrow{\boldsymbol{M M}_{1}}$ and $\overrightarrow{\boldsymbol{M M}_{2}}$ are obtained.

$$
\begin{aligned}
& \overrightarrow{\boldsymbol{M M}_{1}^{m}}=-r \sin \phi\left[\begin{array}{c}
k_{j} \cos \theta_{j} \cos t_{j} \\
\cos \theta_{j} \sin t_{j} \\
\sin \theta_{j}
\end{array}\right]+ \\
& \frac{r \cos \phi}{\sqrt{m_{j}^{\prime 2}+n_{j}^{2}}}\left[\begin{array}{c}
-k_{j}\left(n_{j} \sin t_{j}+m_{j}^{\prime} \sin \theta_{j} \cos t_{j}\right) \\
n_{j} \cos t_{j}-m_{j}^{\prime} \sin \theta_{j} \sin t_{j} \\
m_{j}^{\prime} \cos \theta_{j}
\end{array}\right] \text {, } \\
& \overrightarrow{\boldsymbol{M M}_{2}{ }^{m}}=-r \sin \phi\left[\begin{array}{c}
k_{j} \cos \theta_{j} \cos t_{j} \\
\cos \theta_{j} \sin t_{j} \\
\sin \theta_{j}
\end{array}\right]- \\
& \frac{r \cos \phi}{\sqrt{m_{j}^{\prime 2}+n_{j}^{2}}}\left[\begin{array}{c}
-k_{j}\left(n_{j} \sin t_{j}+m_{j}^{\prime} \sin \theta_{j} \cos t_{j}\right) \\
n_{j} \cos t_{j}-m_{j}^{\prime} \sin \theta_{j} \sin t_{j} \\
m_{j}^{\prime} \cos \theta_{j}
\end{array}\right] .
\end{aligned}
$$

From Eqs. (9), (11) and (22), the first tooth thickness auxiliary curves and the second tooth thickness auxiliary curves were represented as:

$$
\begin{aligned}
\boldsymbol{R}_{j 1 c}^{m} & =\boldsymbol{R}_{j 1}^{m}+\overrightarrow{\boldsymbol{M M}_{1}{ }^{m}}, \\
\boldsymbol{R}_{j 2 c}^{m} & =\boldsymbol{R}_{j 2}^{m}+\boldsymbol{M}_{j 2 j 1}^{m} \cdot \overrightarrow{\boldsymbol{M M}_{2}{ }^{m}},
\end{aligned}
$$

where $\boldsymbol{R}_{11 c}^{m}$ and $\boldsymbol{R}_{12 c}^{m}$ represent the first tooth thickness auxiliary curve and the second tooth thickness auxiliary curve of the driving LG, respectively. $\boldsymbol{R}_{21 c}^{m}$ and $\boldsymbol{R}_{22 c}^{m}$ represent the first tooth thickness auxiliary curve and the second tooth thickness auxiliary curve of the driven LG, respectively.

The analytical expressions of the tooth surfaces $\Sigma_{j 1}^{m}$ and $\Sigma_{j 2}^{m}$ in the coordinate system $o_{m}-x_{m} y_{m} z_{m}$ are also achieved.

$$
\begin{gathered}
\boldsymbol{r}_{j 1}=r \sin \delta\left[\begin{array}{c}
k_{j} \cos \theta_{j} \cos t_{j} \\
\cos \theta_{j} \sin t_{j} \\
\sin \theta_{j}
\end{array}\right]+ \\
\frac{r \cos \delta}{\sqrt{m_{j}^{\prime 2}+n_{j}^{2}}}\left[\begin{array}{c}
-k_{j}\left(n_{j} \sin t_{j}+m_{j}^{\prime} \sin \theta_{j} \cos t_{j}\right) \\
n_{j} \cos t_{j}-m_{j}^{\prime} \sin \theta_{j} \sin t_{j} \\
m_{j}^{\prime} \cos \theta_{j}
\end{array}\right], \\
\boldsymbol{r}_{j 2}=r \sin \delta\left[\begin{array}{c}
k_{j} \cos \theta_{j} \cos t_{j} \\
\cos \theta_{j} \sin t_{j} \\
\sin \theta_{j}
\end{array}\right]- \\
\frac{r \cos \delta}{\sqrt{m_{j}^{\prime 2}+n_{j}^{2}}}\left[\begin{array}{c}
-k_{j}\left(n_{j} \sin t_{j}+m_{j}^{\prime} \sin \theta_{j} \cos t_{j}\right) \\
n_{j} \cos t_{j}-m_{j}^{\prime} \sin \theta_{j} \sin t_{j} \\
m_{j}^{\prime} \cos \theta_{j}
\end{array}\right] .
\end{gathered}
$$

The 'sweep' function in 3D modelling software is usually used to model the line-tooth entity. Firstly, the first contact curve and the first tooth thickness auxiliary curve are plotted by submitting the required parameters into the Eqs. (9) and (16), and a convex arc tooth profile is plotted with a radius of $r$ in a normal plane of the first contact curve. The centre of the arc is located at the first tooth thickness auxiliary curve. Then, the arc tooth profile sweeps along the first contact curve and the first tooth thickness auxiliary curve, taking a role of the trajectory line, by the setting of the tooth profile to be constantly perpendicular to the first contact curve. With the use of 'Swept Blend' to the modelling of line tooth entity in Pro/E, the contact curve should be selected as 'Origin trajectory', along which the tooth profile sweeps, while the corresponding tooth thickness auxiliary curve should be selected as the ' $\mathrm{X}$ vector'. Then, 'Perpendicular to the trajectory' should be selected. In another way, with the use of 'Sweep' in Solidworks, the contact curves should be selected as the 'Path', and the corresponding tooth thickness auxiliary curve should be selected as the ' 1 st guide curve'. By selecting of 'Follow path and 1st guide curve', the twist of the intermediate sections is determined by the vector from the path to the first guide curve, so that the tooth profile remains 
perpendicular to the contact curve. The process above forms a tooth surface containing the first contact curve. Finally, another tooth surface containing the second contact curve is formed by the same method, and the modelling of a line tooth entity is completed.

\section{COMPARISON OF SLIDING RATES}

The relative sliding between a traditional gear pair might cause surface wear and frictional loss, and then the transmission efficiency and fatigue life of the gears are affected. In general, the sliding rates are one of the tribological parameters and subsequently defined and calculated in theory.

Similar to the traditional gear pair, the sliding rates of the LG pair are defined as the limit value of the ratio of the lengths difference between two relative arcs divided by the length of the given arc [24], they are expressed as Eq. (26).

$$
\begin{gathered}
\sigma_{1}=1-\frac{\sqrt{\left(x_{M}^{\prime(2)}\right)^{2}+\left(y_{M}^{\prime(2)}\right)^{2}+\left(z_{M}^{\prime(2)}\right)^{2}}}{\sqrt{\left(x_{M}^{\prime(1)}\right)^{2}+\left(y_{M}^{\prime(1)}\right)^{2}+\left(z_{M}^{\prime(1)}\right)^{2}}}, \\
\sigma_{2}=1-\frac{\sqrt{\left(x_{M}^{\prime(1)}\right)^{2}+\left(y_{M}^{\prime(1)}\right)^{2}+\left(z_{M}^{\prime(1)}\right)^{2}}}{\sqrt{\left(x_{M}^{(2)}\right)^{2}+\left(y_{M}^{\prime(2)}\right)^{2}+\left(z_{M}^{\prime(2)}\right)^{2}}},
\end{gathered}
$$

where $\sigma_{1}$ and $\sigma_{2}$ represent the sliding rates of the driving $L G$ and the driven $L G$, respectively, $x_{M}^{(j)}=\frac{d x_{j}}{d t}, y_{M}^{(j)}=\frac{d y_{j}}{d t}, z_{M}^{(j)}=\frac{d z_{j}}{d t}, j=1,2$.

Taking three couples of LG with vertical cross axes as design examples, the values of various parameters of LG pairs are shown in Table 1. The driving contact curve of the LG pair No. 1 is a circular helix, and the driving contact curves of the LG pair No. 2 and the LG pair No. 3 are conic helixes with a half cone angle of $7^{\circ}$. The heights of the driving LGs are all $16 \mathrm{~mm}$ for three examples. The driving contact curves and the driven contact curves can be derived from Eq. (9).

Table 1. The various parameters of designed the LG pair

\begin{tabular}{ccccccc}
\hline No. & $m_{1}[\mathrm{~mm}]$ & $n_{1}[\mathrm{~mm}]$ & $\theta_{1}\left[^{\circ}\right]$ & $m_{2}[\mathrm{~mm}]$ & $i$ & $\theta_{0}\left[^{\circ}\right]$ \\
\hline 1 & 2.5 & 4 & 0 & 12 & 8 & 90 \\
2 & 1.5 & 4 & 7 & 12 & 8 & 90 \\
3 & 1.8 & 4 & 7 & 12 & 8 & 90 \\
\hline
\end{tabular}

The 3D solid models of the LG pairs No. 1 and No. 2 are shown in Fig. 6, with the different shapes of the driving $L G$. a)

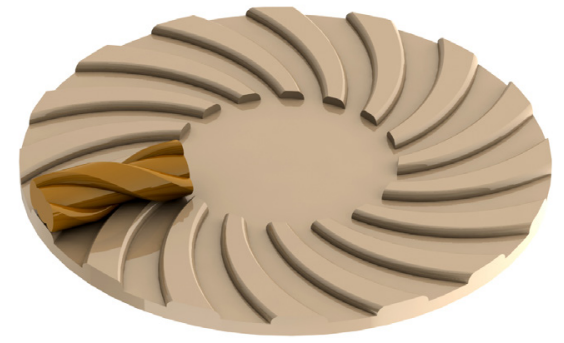

b)

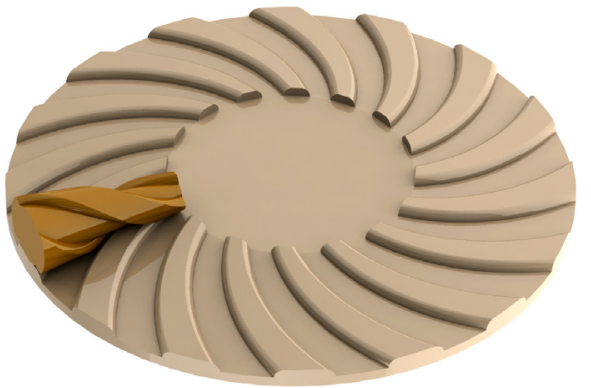

Fig. 6. 3D solid model; a) the LG pair No. 1, b) the LG pair No. 2

From Eqs. (9) and (26), the sliding rates of contact curves of three LG pairs are obtained and plotted with Wolfram Mathematica, as shown in Fig. 7.

a)
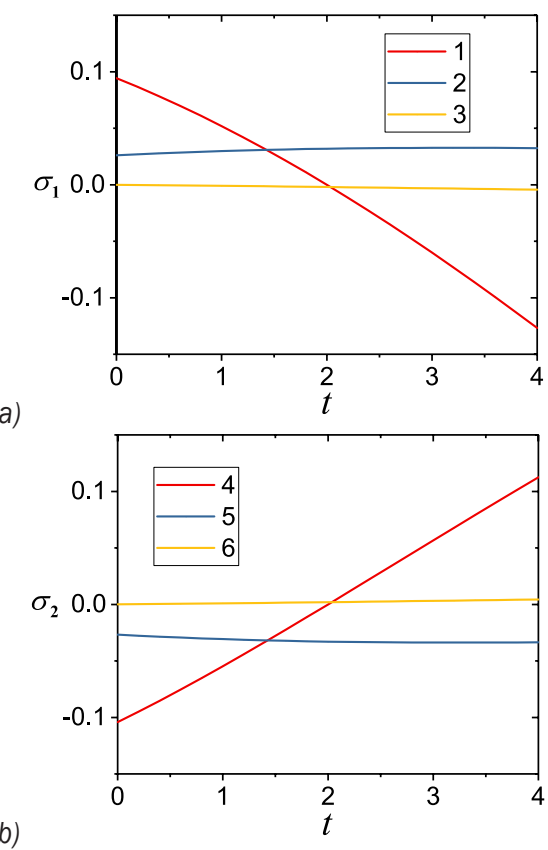

Fig. 7. Sliding rates of the $L G$ pair No. 1-3; a) $\left.\sigma_{1} v s t, b\right) \sigma_{2}$ vs $t$ (1- $\sigma_{1}$ of the $L G$ pair No. 1; 2- $\sigma_{1}$ of the $L G$ pair No. 2; 3- $\sigma_{1}$ of the $L G$ pair No. 3; $4-\sigma_{2}$ of the $L G$ pair No. $1 ; 5-\sigma_{2}$ of the $L G$ pair No. 2; $6-\sigma_{2}$ of the $L G$ pair No. 3)

From Fig. 7, the sliding rates of the LG pairs, of which the relative position parameters are limited, can be controlled by changing the shape of the driving 
contact curve. When the driving contact curve is designed by a certain conic helix, the absolute value of sliding rates can be significantly decreased.

\section{A KINEMATICS EXPERIMENT}

A 3D printing technology, stereolithography appearance (SLA), was adopted to manufacture the samples of the designed LG pair. The surface profile accuracy of the process is $0.025 \mathrm{~mm}$. Fig. 8 shows the prototypes of the manufactured LG pair No. 2; the driving LG is on the left, and the driven LG is on the right.

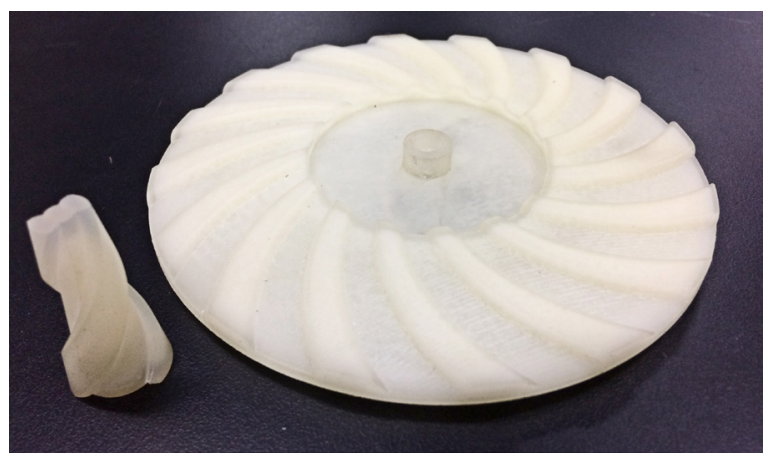

Fig. 8. The prototypes of the LG pair No. 2

To measure the transmission ratio of the designed LG pair, a kinematics experiment was conducted by use of a test rig with a 4-DOF positioning table, as shown in Fig. 9.

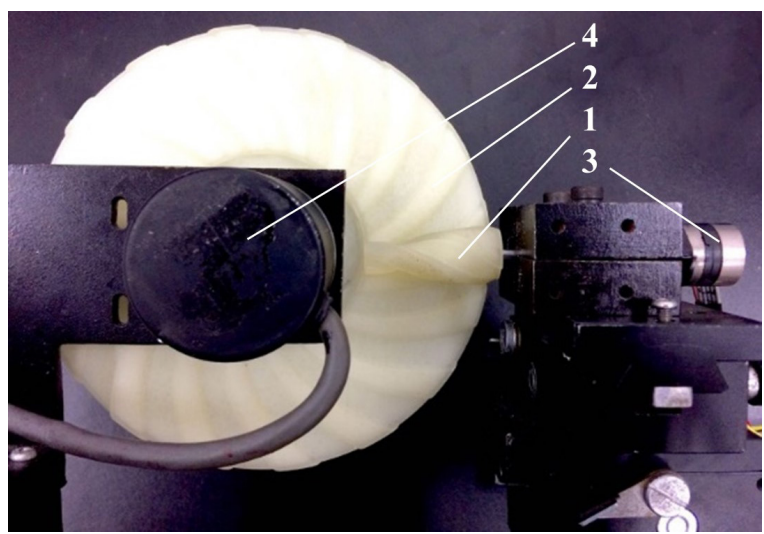

Fig. 9. The test rig (1-the driving LG;2-the driven LG; 3-a DC motor with the encoder;4-a encoder)

The driving LG and the driven LG were fixed with encoders and the Decklink SDI to collect their angular rotations in the same time interval. The sampling interval in this experiment was set as $150 \mathrm{~ms}$. Then the instant angular velocity was calculated. The ratio of the instant angular velocity of the driven $L G$ to the driving $L G$ is defined as the instant transmission ratio of the prototype of the designed LGs, as shown in Fig. 10.

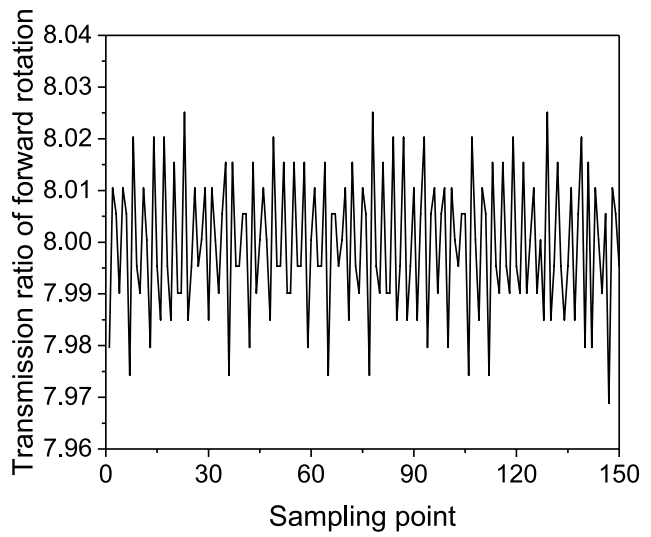

Fig. 10. The instant transmission ratio; a) under the forward rotation and b) under the reverse rotation

As shown in Fig. 10, the instant transmission ratios are fluctuating around the theoretical design value of 8 , which means the results of the kinematics experiment are consistent with the desired value. The relative error is less than $0.01 \%$. The maximum instant relative errors under the forward rotation and under the reverse rotation are $0.314 \%$ and $0.389 \%$, respectively, which may come from the following aspects: manufacturing error of the contact curves, installing error and positioning error, and data errors in collecting and calculating covering original data error, truncation error and round-off error.

\section{CONCLUSION}

This paper proposed the design methodology of coplanar axes line gear with the controllable sliding rate for non-backlash forward-reverse transmission. The main conclusions include: 
(1) The driving contact curve of an LG pair with coplanar axes was extended from a circular helix to a conic helix, and the driven contact curve was derived on the basis of a given driving contact curve. This process provides a new method to control the sliding rates of the contact curves. The design examples show that the LG pair, of which the driving contact curves are conic helixes, possess better sliding rates.

(2) There are two contact curves lying on two sides of a line tooth, and the two couples of contact curves that lie along the driving line tooth and the driven line tooth are two couples of conjugate curves. A line tooth entity, attachment to the wheel body, is formed by a normal arc profile moving along the contact curves and the tooth thickness auxiliary curves. The analytical expressions of spatial cylindrical tooth surfaces are also achieved. The proposed LG pair could be capable of achieving forward-reverse transmission continuously and smoothly without backlash.

However, some issues for the proposed LG pair remain to be studied, covering that selection criterion of the geometric parameters, an experimental measure method of the sliding rates, the failure criteria, bending and contact strength, the efficiency of transmission, etc.

\section{ACKNOWLEDGEMENT}

The authors gratefully acknowledge the support from National Natural Science Foundation of China (No. 51575191), China Postdoctoral Science Foundation (No. 2017M612652) and Central university basic scientific research service fee (No. 2017B0071).

\section{NOMENCLATURE}

$v_{12}$ relative velocity at the meshing point between the driving line tooth and the driven line tooth, $[\mathrm{m} / \mathrm{s}]$

$\boldsymbol{\beta} \quad$ unit principal normal vector of the driving contact curve at the meshing point,

$o_{0}-x_{0} y_{0} z_{0}, o_{1}-x_{1} y_{1} z_{1}$ fixed coordinate system and follow-up coordinate system of driving LG,

$o_{p}-x_{p} y_{p} z_{p}, o_{2}-x_{2} y_{2} z_{2}$ fixed coordinate system and follow-up coordinate system of driven LG,

$\theta_{0} \quad$ shaft angle between driving LG and driven $\mathrm{LG},\left[^{\circ}\right]$

$a$ distances from point $o_{0}$ to $o_{p},[\mathrm{~mm}]$

$t_{1}, t_{2} \quad$ parameter variable of driving contact curves and driven contact curves,

$i \quad$ transmission ratio,
$N_{1}, N_{2}$ tooth numbers of driving $\mathrm{LG}$ and driven LG,

$m_{1}, n_{1}, m_{2}, n_{2}$ conical parameters of driving contact curves and driven contact curves, [mm]

$\theta_{1}, \theta_{2}$ half cone angles of driving contact curves and driven contact curves, $\left[^{\circ}\right]$

$s_{1}, s_{2}$ line tooth thicknesses of driving LG and driven LG, [mm]

$e_{1}, e_{2}$ space widths of driving LG and driven LG, [mm]

$\varphi_{1 F R}, \varphi_{2 F R}$ phase differences between first contact curve and second contact curve,

$\alpha \quad$ unit tangent vector of the driving contact curve at the meshing point,

$\overrightarrow{\boldsymbol{M M}_{1}}, \overrightarrow{\boldsymbol{M M}_{2}}$ Contact vector from $M$ to $M_{1}$ and $\mathrm{M}_{2}$,

$r \quad$ arc radius of tooth profile, [mm]

$\boldsymbol{R}_{11}^{m}, \boldsymbol{R}_{12}^{m}, \boldsymbol{R}_{21}^{m}, \boldsymbol{R}_{22}^{m} \quad$ first contact curve and second contact curve of driving LG and driven LG,

$\boldsymbol{R}_{11 c}^{m}, \quad \boldsymbol{R}_{12 c}^{m}, \quad \boldsymbol{R}_{21 c}^{m}, \quad \boldsymbol{R}_{22 c}^{m} \quad$ first and second tooth thickness auxiliary curves of driving LG and driven $L G$,

$\Sigma_{11}^{m}, \Sigma_{12}^{m}, \Sigma_{21}^{m}, \Sigma_{22}^{m}$ the analytical expressions of tooth surfaces of driving LG and driven LG,

$\sigma_{1}, \sigma_{2}$ sliding rates of driving contact curve and driven contact curve.

\section{REFERENCES}

[1] Litvin, F.L., Fuentes, A. (2004). Gear Geometry and Applied Theory (2nd ed.), Cambridge University Press, New York, DOI:10.1017/СB09780511547126.

[2] Osplack, J.J., Beam, A.S. (1956). Conical Involute Gearing. US, US2747424.

[3] Brauer, J. (2002). Analytical geometry of straight conical involute gears. Mechanism and Machine Theory, vol. 37, no. 1, p. 127-141, DOI:10.1016/S0094-114X(01)00062-3.

[4] Liu, C.C. Tsay, C.B. (2002). Contact characteristics of beveloid gears. Mechanism \& Machine Theory, vol. 37, no. 4, p. 333350, D0I:10.1016/S0094-114X(01)00082-9.

[5] Staniek, R. (2011). Shaping of face toothing in flat spiroid gears. Strojniški vestnik - Journal of Mechanical Engineering, vol. 57, no. 1, p. 47-54, Dol:10.5545/sv-jme.2010.093.

[6] Sugimoto, M., Maruyama, N., Nakayama, A., Hitomi, N. (1991). Effect of tooth contact and gear dimensions on transmission errors of loaded hypoid gears. Journal of Mechanical Design, vol. 113, no. 2, p. 182-187, Dol:10.1115/1.2912767.

[7] Litvin, F.L., Vecchiato, D., Gurovich, E., Fuentes, A., GonzalezPerez, I., Hayasaka, K., Yukishima, K. (2005). Computerized developments in design, generation, simulation of meshing, and stress analysis of gear drives. Meccanica, vol. 40, no. 3, p. 291-323, DOI:10.1007/s11012-005-4020-y.

[8] Tsai, Y.C., Hsu, W.Y. (2008). The study on the design of spiral bevel gear sets with circular-arc contact paths and tooth 
profiles. Mechanism and Machine Theory, vol. 43, no. 9, p. 1158-1174, DOI:10.1016/j.mechmachtheory.2007.08.004.

[9] Alves, J.T., Guingand, M., de Vaujany, J.P. (2013). Designing and manufacturing spiral bevel gears using 5-axis computer numerical control (CNC) milling machines. Journal of Mechanical Design, vol. 135, no. 2, p. 024502, DOI:10.1115/1.4023153.

[10] Simon, V.V. (2015). Design and manufacture of spiral bevel gears with reduced transmission errors. Journal of Mechanical Design, vol. 131, no. 4, p. 041007-041017, DOI:10.1115/1.3087540.

[11] Chen, Y.Z. (2014). Line Gear. Science Press, Beijing. (in Chinese)

[12] Chen, Y.Z., Xing, G.Q., Peng, X.F. (2007). The space curve mesh equation and its kinematics experiment. 12th IFToMM World Congress, Besançon.

[13] Chen, Y.Z., Xiang, X.Y., Luo, L. (2009). A corrected equation of space curve meshing. Mechanism and Machine Theory, vol. 44, no. 7, p. 1348-1359, D0l:10.1016/j. mechmachtheory.2008.11.001.

[14] Ding, J., Chen, Y.Z., Lv, Y.L. (2012). Design of Space-Curve Meshing-Wheels with Unequal Tine Radii. Strojniški vestnik - Journal of Mechanical Engineering, vol. 58, no. 11, p. 633641, DOl:10.5545/sv-jme.2012.493.

[15] Chen, Y.Z., Lv, Y.L., Ding, J., Chen, Z. (2013). Fundamental design equations for space curve meshing skew gear mechanism. Mechanism and Machine Theory, vol. 70, p. 175188, D0I:10.1016/j.mechmachtheory.2013.07.004.

[16] Chen, Y.Z., Luo, L., Hu, Q. (2009). The contact ratio of a space-curve meshing-wheel transmission mechanism. Journal of Mechanical Design, vol. 131, no. 7, p. 074501, DOI:10.1115/1.3116343.
[17] Lv, Y.L., Chen, Y.Z., Cui, X.Y. (2015). A contact ratio and interference-proof conditions for a skew line gear mechanism. Transactions of the Canadian Society for Mechanical Engineering, vol. 39, no. 3, p. 647-656, D0l:10.1139/ tcsme-2015-0051.

[18] Chen, Y. Z., Yao, L. (2016). Design formulae for a concave convex arc line gear mechanism. Mechanical Sciences, vol. 7 , no. 2, p. 209-218, Dol:10.5194/ms-7-209-2016.

[19] Wu, X.T. (2009). Principle of Gearing. Jiaotong University Press, Xi'an. (in Chinese)

[20] Li, T., Pan, C., Gao, F., Wang, X. (2012). Research on sliding ratios of conjugate surfaces of two degrees of freedom meshing transmission of spherical gear pair. Journal of Mechanical Design, vol. 134 , no. 9, p. 091002, DOI:10.1115/1.4006322.

[21] Narayanan, G. (2016). Effect of sliding friction on spline surface failure under misaligned condition in aero engines. International Journal of Structural Integrity, vol. 7, no. 5, DOI:10.1108/IJSI-07-2015-0024.

[22] Qian, S., Guo, D., Liu, S., Lu, X. (2011). Experimental investigation of lubrication failure of polyalphaolefin oil film at high slide/roll ratios. Tribology Letters, vol. 44, no. 2, p. 107115, D0I:10.1007/s11249-011-9820-8.

[23] Ciulli, E., Stadler, K., Draexl, T. (2009). The influence of the slide-to-roll ratio on the friction coefficient and film thickness of EHD point contacts under steady state and transient conditions. Tribology International, vol. 42, no. 4, p. 526-534, D0l:10.1016/j.triboint.2008.04.005.

[24] Ding, J., Chen, Y. Z., Lv, Y. L., Song, C. (2014). Positionparameter selection criterion for a helix-curve meshing-wheel mechanism based on sliding rates. Strojniški vestnik - Journal of Mechanical Engineering, vol. 60, no. 9, p. 561-570, D0I:10.5545/sv-jme.2013.1574. 